\title{
TH1B-T5-4
}

\section{Optical Biopsy of Liver Fibrosis using Multiphoton Microscopy}

\author{
Yuan Liu ${ }^{1}$, Hsuan-Shu Lee ${ }^{2}$, Hsiao-Ching Chen ${ }^{2}$, Ling-Ling Chiou ${ }^{2}$, Guan-Tarn Huang ${ }^{2}$, \\ Wen Lo ${ }^{1}$, Chen-Yuan Dong ${ }^{1 *}$ \\ 'Department of Physics, National Taiwan University, Taipei, Taiwan 106 \\ ${ }^{2}$ Department of Internal Medicine, National Taiwan University Hospital, Taipei, Taiwan 100 \\ 886-2-33665196, 886-2-3665244, circle925@yahoo.com.tw \\ *To whom correspondence should be addressed: cydong@phys.ntu.edu.tw
}

\begin{abstract}
In this work, we apply multiphoton microscopy in diagnosing toxin (CCl4) induced liver fibrosis in mice. We found that multiphoton induced spectroscopic signatures may be used to identify stellate cell proliferation and necrotic sites. Our study suggests that multiphoton imaging may be developed into an effective diagnosis tool for
\end{abstract} liver fibrosis in vivo.

\section{Introduction}

Liver diseases are serious problems to human health. Conventional histological procedures limit the ability to diagnose liver tissues in living specimens, and the development of intravital imaging techniques is invaluable in following liver physiology in vivo. The purpose of this study is to test the capabilities of multiphoton imaging in the diagnosis of liver fibrosis in vivo, thus, lead to early detection and treatment.

\section{Materials and Methods}

We used the $780 \mathrm{~nm}$ output of a femtosecond, titanium- sapphire laser as the excitation source. The laser is scanned by an $x-y$ scanning system. After being processed by the optical instruments and the microscope, images are collected by the PMT and recorded by the computer.

Liver fibrosis was induced from male $\mathrm{BALB} / \mathrm{c}$ mice injected with $\mathrm{CCl}_{4}$. Starting at 6 weeks of ages, the mice were given intra-peritoneal injections of 1:9 volumetric ratios of $\mathrm{CCl}_{4}$ and olive oil. Twice weekly, the dosage of 1 $\mu \mathrm{CCl}_{4}$ per gram of mouse body mass was applied. The mice were sacrificed 24 hours after the final injection and the left medial lobe of the liver was removed to be imaged from the surface using the multiphoton microscope described above.

\section{Results and Conclusions}

Multiphoton images of normal and mice livers after 1,2, and 3 weeks of $\mathrm{CCl}_{4}$ induction are shown in Figs. 1-4(next page). Distribution of hepatocytes (elliptically enclosed regions) and stellate cells in normal mouse liver and those treated with 1,2 , and 3 weeks of $\mathrm{CCl} 4$ (yellow arrow: a stellate cell) are identifiable.

Left: magnified multiphoton image. Center: large area multiphoton scan $(1.27 \mathrm{~mm}$ by $1.21 \mathrm{~mm})$. Right: histologicalimages (hepatocyte nuclei: purple; stellate cells: red). The scale bar is $100 \mu \mathrm{m}$.

We have shown that multiphoton microscopy is capable of identifying liver damage in mice following the treatment of $\mathrm{CCl}_{4}$. Cell necrosis and the proliferation of stellate cell at the damaged sites can be identified under multiphoton imaging. Our results suggest that multiphoton imaging may be developed into effective diagnostic tool for liver fibrosis in vivo.

\section{References}

1. W. Denk, J. H. Strickler, and W. W. Webb, "2 -photon laser scanning fluorescencemicroscopy," Science 248 (4951), 73 (1990).

2. P. T. C. So, C. Y. Dong, B. R. Masters, and K. M. Berland, "Two-photon excitation fluorescence microscopy," Annual Review of Biomedical Engineering 2, 399(2000).

3. B. Yu, C. Y. Dong, P. T. C. So, D. Blankschtein, and R. Langer, "In vitro visualization and quantification of oleic acid induced changes in transdermal transport using two-photon fluorescence microscopy," Journal of Investigative Dermatology 117(l), 16 (2001).

4. Y. Sun, J. W. Su, W. Lo, S. J. Lin, S. H. Jee, and C. Y. Dong, "Multiphoton polarization imaging of the stratum corneum and the dermis in ex-vivo human skin," Optics Express 11(25), 3377 (2003).

5. Y. Sun, W. Lo, S. J. Lin, S. H. Jee, and C. Y. Dong, "Multiphoton polarization and generalized polarization (GP) microscopy reveals oleic acid induced structural changes in intercellular lipid layers of the skin," Optics Letters, Accepted.

6. E. B. Brown, R. B. Campbell, Y. Tsuzuki, L. Xu, P. Carmeliet, D. Fukumura, and R. K. Jain. "In vivo measurement of gene expression, angiogenesis and physiological function in tumors using multiphoton laser scanning microscopy," Nature Medicine 7(7), 864 (2001). 
TH1B-T5-4
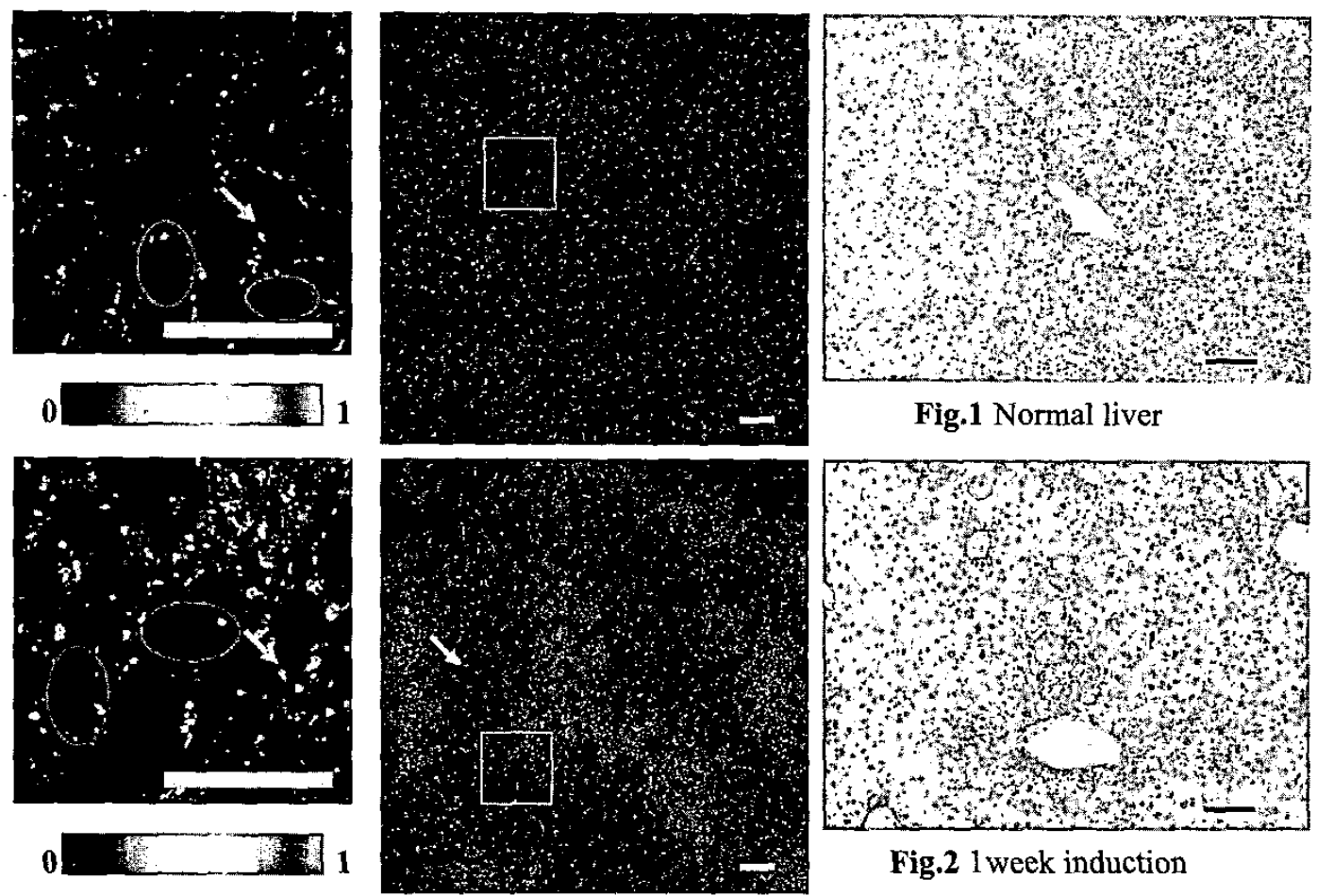

Fig.1 Normal liver

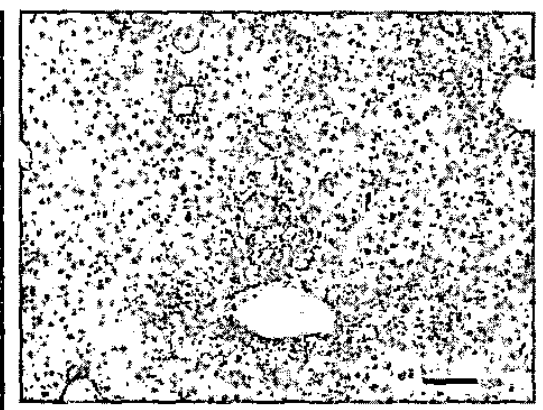

Fig.2 1week induction
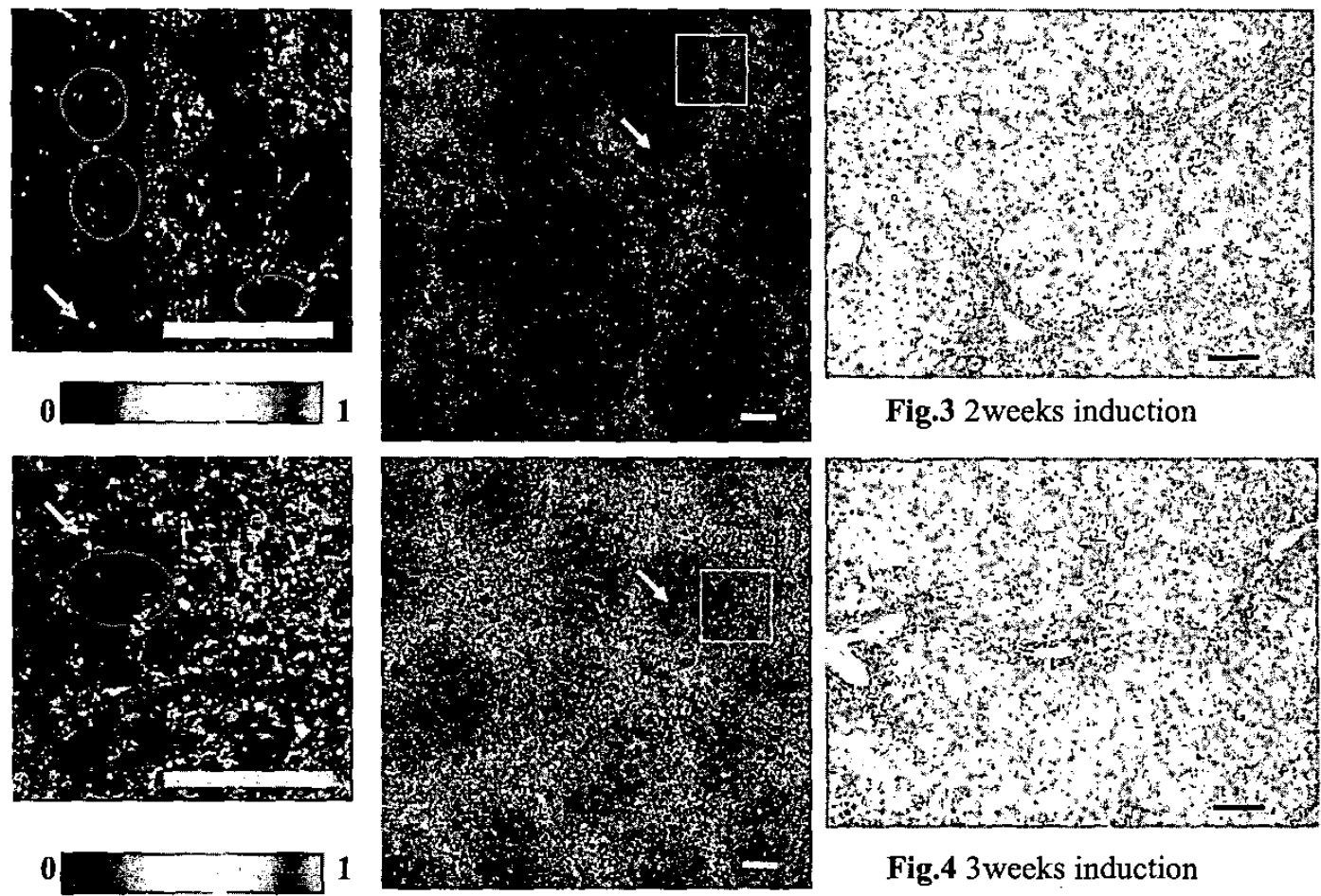

Fig.3 2weeks induction

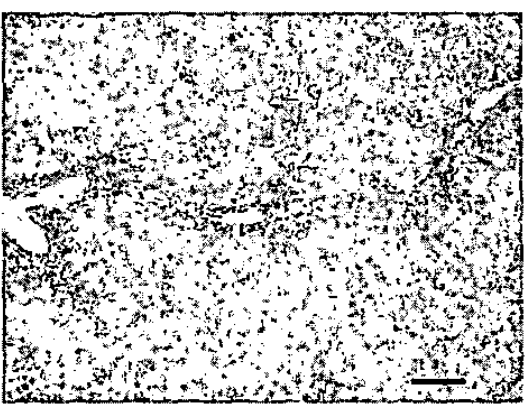

Fig.4 3weeks induction 